\title{
ON CONJUGACY CLASSES OF ELEMENTS OF FINITE ORDER IN COMPACT OR COMPLEX SEMISIMPLE LIE GROUPS
}

\author{
DRAGOMIR Ž. DJOKOVIĆ
}

\begin{abstract}
If $K$ is a connected compact Lie group with simple Lie algebra and if $k$ is an integer relatively prime to the order of the Weyl group $W$ of $K$ then the number $\nu(K, k)$ of conjugacy classes of $K$ consisting of elements $x$ satisfying $x^{k}=1$ is given by
\end{abstract}

$$
\nu(K, k)=\prod_{i=1}^{l} \frac{m_{i}+k}{m_{i}+1},
$$

where $l$ is the rank of $K$ and $m_{1}, \ldots, m_{l}$ are the exponents of $W$. If $G$ is the complexification of $K$ then we have $\nu(G, k)=\nu(K, k)$ without any restriction on $k$.

Results and proofs. If $G$ is a group and $k$ a positive integer we write $G(k)=$ $\left\{x \in G \mid x^{k}=1\right\}$. We denote by $\nu(G, k)$ the number of conjugacy classes of $G$ contained in $G(k)$. (In our cases $\nu(G, k)$ will be finite.)

LEMMA 1. Let $Z_{1}$ be a finite subgroup of the center of $G$. If $k$ and $\left|Z_{1}\right|$ are relatively prime then the canonical map $G \rightarrow G / Z_{1}$ induces a bijection $G(k) \rightarrow\left(G / Z_{1}\right)(k)$ and $\nu(G, k)=\nu\left(G / Z_{1}, k\right)$.

Proof. Let $x, y \in G(k)$ and assume that $x Z_{1}=y Z_{1}$. Then $y=x z$ for some $z \in Z_{1}$. Hence $1=y^{k}=(x z)^{k}=z^{k}$. Since $k$ and $\left|Z_{1}\right|$ are relatively prime, we have $z=1$, and so $x=y$.

Now let $x \in G$ be such that $x Z_{1} \in\left(G / Z_{1}\right)(k)$, i.e., $x^{k} \in Z_{1}$. Since $k$ and $\left|Z_{1}\right|$ are relatively prime, there exists $z \in Z_{1}$ such that $x^{k}=z^{k}$. Then $y=x z^{-1} \in G(k)$ and $y Z_{1}=x Z_{1}$. Thus we have shown that $G(k) \rightarrow\left(G / Z_{1}\right)(k)$ is a bijection. The second assertion now follows immediately.

From now on let $G$ be a connected complex semisimple Lie group, $g$ its Lie algebra, $l$ the rank of $g, h$ its Cartan subalgebra, $H$ the corresponding Cartan subgroup of $G, N$ the normalizer of $H$ in $G$, and $W=N / H$ the Weyl group of $(g, h)$. By $P$ we denote the group of weights of $(g, h)$ and by $Q$ the subgroup of $P$ of radical weights. Both $P$ and $Q$ are free abelian groups of rank $l$, and $Q$ is generated by the root system $\Sigma$ of $(g, h)$. The group $H$ is an algebraic torus, i.e., $H$ is isomorphic to the product of $l$ copies of the group $C^{*}$ of nonzero complex numbers. The exponential map $\exp _{H}: h \rightarrow H$ is a surjective homomorphism. The

Received by the editors September 20, 1979. Presented at the 53rd Ontario Math. Meeting, Toronto, November 25, 1979.

AMS (MOS) subject classifications (1970). Primary 22E10; Secondary 20 G20.

Key words and phrases. Semisimple complex Lie group, compact semisimple group, Weyl group, conjugacy classes, orbits, exponents of the Weyl group.

${ }^{1}$ Partially supported by NRC Grant A-5285. 
kernel $L_{G}$ of the homomorphism $f: h \rightarrow H$ defined by $f(X)=\exp _{H}(2 \pi i X)$ is also a free abelian group of rank $l$ which is generated by a basis of $h$ (considered as a complex vector space). Let $P_{G}$ be the subgroup of the dual space $h^{*}$ consisting of the elements $\alpha$ such that $\alpha(X)$ is an integer for all $X \in L_{G}$. Then we have $Q \subset P_{G} \subset P$. We fix a positive integer $k$. The Weyl group $W$ acts on $h$ and $h^{*}$ and $P_{G}$ is stable under this action. Hence $W$ also acts on the finite abelian group $P_{G} / k P_{G}$. (Note that this abelian group is a direct product of $l$ cyclic groups of order $k$.) We recall also that the order $e$ of $P / Q$ is called the connection index of $g$ (or $W$ ).

Our first result is the following.

THEOREM 2. Let $K$ be a maximal compact subgroup of $G$. Then $\nu(G, k)=\nu(K, k)$ and this number is also equal to the number of orbits of $W$ in $P_{G} / k P_{G}$.

Proof. Let $T$ be the (unique) maximal compact subgroup of $H$. Since all maximal compact subgroups of $G$ are conjugate in $G$ we may assume that $T \subset K$. Then $T$ is a maximal torus of $K$. Every $x \in G(k)$ is conjugate to some $y \in K$. Since $K$ is connected, $y$ is conjugate in $K$ to some element of $T$. Hence every conjugacy class of $G$ which is contained in $G(k)$ meets $H(k)$. On the other hand two elements of $H$ are conjugate in $G$ iff they belong to the same orbit of $W$ in $H$. This shows that the inclusion map $H(k) \rightarrow G(k)$ induces a bijection from the set of $W$-orbits in $H(k)$ to the set of $G$-conjugacy classes contained in $G(k)$. Similarly, the inclusion map $H(k)=T(k) \rightarrow K(k)$ induces a bijection from the set of $W$-orbits in $H(k)$ to the set of $K$-conjugacy classes contained in $K(k)$. In particular, we have $\nu(G, K)=$ $\nu(K, k)$.

The epimorphism $f: h \rightarrow H$ induces a bijection between the set of $W$-orbits in $\left(k^{-1} L_{G}\right) / L_{G}$ and the set of $W$-orbits in $H(k)$. Finally, by duality, the number of $W$-orbits in $\left(k^{-1} L_{G}\right) / L_{G}$ is equal to the number of $W$-orbits in $P_{G} / k P_{G}$. This completes the proof.

Now let us assume that $k$ and $|W|$ are relatively prime. Let $Z$ be the center of $G$. Then it is easy to check that every prime divisor of $|Z|$ also divides $|W|$. Hence $k$ and $|Z|$ are also relatively prime. By Lemma 1 we have then $\nu(G, k)=\nu(G / Z, k)$. Thus we may assume that $G$ is the adjoint group. Then $G$ is a product of simple complex Lie groups $G_{1}, \ldots, G_{r}$. Consequently we have

$$
\nu(G, k)=\prod_{i=1}^{r} \nu\left(G_{i}, k\right) .
$$

This reduces the problem of computing $\nu(G, k)$ to the case when $G$ is a simple complex Lie group (with trivial center). In that case the answer is given in the following theorem.

THEOREM 3. Assume that $G$ is the adjoint group, $g$ is simple, and that $k$ and $W$ are relatively prime. Then we have

$$
\nu(G, k)=\prod_{i=1}^{l} \frac{m_{i}+k}{m_{i}+1}
$$

where $m_{1}, \ldots, m_{l}$ are the exponents of $W .($ See $[1, p .118]$. 
Proof. In this case we have $P_{G}=Q$. By Theorem $2, \nu(G, k)$ is equal to the number of orbits of $W$ in $Q / k Q$.

For each root $\alpha \in \Sigma$ let $s_{\alpha} \in W$ be the corresponding reflection. A root system $\Sigma_{1} \subset \Sigma$ is closed in $\Sigma$ if $\alpha, \beta \in \Sigma_{1}$ and $\alpha+\beta \in \Sigma$ imply that $\alpha+\beta \in \Sigma_{1}$. A subgroup $W_{1} \subset W$ is called a Weyl subgroup if there exists a closed subsystem $\Sigma_{1} \subset \Sigma$ such that $W_{1}$ is generated by the reflections $s_{\alpha}$ for all $\alpha \in \Sigma_{1}$. Then the set of all $\alpha \in \Sigma$ such that $s_{\alpha} \in W_{1}$ is a closed subsystem containing $\Sigma_{1}$. Hence without loss of generality we may assume that $\Sigma_{1}=\left\{\alpha \in \Sigma \mid s_{\alpha} \in W_{1}\right\}$.

Fix $w \in W$ and let $W_{1}$ be a minimal Weyl subgroup of $W$ containing $w$. Define $\Sigma_{1}$ as above and let

$$
\Sigma_{1}=\Sigma_{11} \cup \cdots \cup \Sigma_{1 r}
$$

be the decomposition of $\Sigma_{1}$ into irreducible root systems. Then the real vector subspace of $h^{*}$ spanned by $\Sigma_{1}$ admits a direct decomposition

$$
V_{1}=V_{11}+\cdots+V_{1 r}
$$

where $V_{1 i}$ is spanned by $\Sigma_{1 i}$. This leads to the corresponding direct decomposition of the group $W_{1}$ :

$$
W_{1}=W_{11} \times \cdots \times W_{1 r},
$$

where $W_{1 i}$ is generated by the reflections $s_{\alpha}$ for $\alpha \in \Sigma_{1 i}$. Let $w=w_{1} \ldots w_{r}$ be the corresponding decomposition of the element $w$. By minimality of $W_{1}$, the element $w_{i} \in W_{1 i}(i=1, \ldots, r)$ is not contained in any proper Weyl subgroup of $W_{1 i}$. By [4, Corollary 8.3] we have $\operatorname{det}\left(w_{i}-1\right)= \pm e_{i}$ where $e_{i}$ is the connection index of $W_{1 i}$, and $w_{i}$ is considered as acting in $V_{1 i}$. Hence

$$
\operatorname{det}\left(\left.w\right|_{V_{1}}-1\right)= \pm e_{1} \cdots e_{r}
$$

Since $e_{i}$ divides $\left|W_{1 i}\right|$, and the latter divides $|W|$, it follows that $k$ and the above determinant are relatively prime.

Let $m$ be the multiplicity of the eigenvalue 1 of $w$. Thus $\operatorname{dim} V_{i}=l-m$. By [2, Theorem III.12, p. 50] there exists a basis of $Q$ with respect to which the matrix of $w$ is an integral $l$ by $l$ matrix of the form

$$
\left(\begin{array}{ll}
A & B \\
0 & C
\end{array}\right)
$$

where $A$ is an upper triangular $m$ by $m$ matrix with ones on the diagonal. Since $w$ has finite order, $A$ must be the identity matrix. Since

$$
\operatorname{det}(C-I)=\operatorname{det}\left(\left.w\right|_{V_{1}}-1\right)
$$

the matrix $C-I$ is invertible when considered as a matrix over the residue ring $\mathbf{Z} / k \mathbf{Z}$. Consequently, the number of elements of $Q / k Q$ fixed by $w$ is equal to $k^{m}$.

Let $g_{m}$ be the number of elements $w \in W$ such that 1 is an eigenvalue of $w$ of multiplicity $m$. By a theorem of Solomon [3] we have the identity

$$
\left(m_{1}+t\right) \cdots\left(m_{l}+t\right)=g_{0}+g_{1} t+\cdots+g_{l} t^{l}
$$


Hence $|W|=\left(m_{1}+1\right) \ldots\left(m_{l}+1\right)$ and the number of orbits of $W$ in $Q / k Q$ is equal to

$$
\frac{1}{|W|} \sum_{m=0}^{l} g_{m} k^{m}=\prod_{i=1}^{l} \frac{m_{i}+k}{m_{i}+1} .
$$

This completes the proof of the theorem.

Remarks. 1. The equality $\nu(G, k)=\nu(K, k)$ from Theorem 1 is in fact valid for any compact Lie group $K$ (not necessarily semisimple nor connected) and its complexification $G$.

2. If $\nu(G, d)$ is known for all divisors $d$ of $k$ then by using Inclusion-Exclusion Principle one can easily compute the number of conjugacy classes of $G$ consisting of elements of order $k$.

\section{REFERENCES}

1. N. Bourbaki, Groupes et algèbres de Lie, Hermann, Paris, 1968, Chapters 4-6.

2. M. Newman, Integral matrices, Academic Press, New York, 1972.

3. L. Solomon, Invariants of finite reflection groups, Nagoya Math. J. 22 (1963), 57-64.

4. T. A. Springer, Regular elements of finite reflection groups, Invent. Math. 25 (1974), 159-198.

Department of Pure Mathematics, University of Waterloo, Waterloo, Ontario, Canada N2L $3 \mathrm{Gl}$ 